\title{
Decision Tree Model for Predicting Hospice Palliative Care Use in Terminal Cancer Patients
}

\author{
Hee-Ja Lee, R.N., M.S., Im-II Na, M.D.* and Kyung-Ah Kang, R.N., Ph.D. ${ }^{\dagger}$ \\ Special Nursing Team, Korea Institute of Radiological \& Medical Sciences, "Hospice Care Center, Korea Institute of Radiological \\ $\&$ Medical Sciences, ${ }^{\dagger}$ College of Nursing, Sahmyook University, Seoul, Korea
}

Purpose: This study attempted to develop clinical guidelines to help patients use hospice and palliative care (HPC) at an appropriate time after writing physician orders for lifesustaining treatment (POLST) by identifying the characteristics of HPC use of patients with terminal cancer. Methods: This retrospective study was conducted to understand the characteristics of HPC use of patients with terminal cancer through decision tree analysis. The participants were 394 terminal cancer patients who were hospitalized at a cancer-specialized hospital in Seoul, South Korea and wrote POLST from January 1, 2019 to March 31, 2021. Results: The predictive model for the characteristics of HPC use showed three main nodes (living together, pain control, and period to death after writing POLST). The decision tree analysis of HPC use by terminal cancer patients showed that the most likely group to use HPC use was terminal cancer patients who had a cohabitant, received pain control, and died 2 months or more after writing a POLST. The probability of HPC usage rate in this group was $87.5 \%$. The next most likely group to use HPC had a cohabitant and received pain control; $64.8 \%$ of this group used HPC. Finally, $55.1 \%$ of participants who had a cohabitant used HPC, which was a significantly higher proportion than that of participants who did not have a cohabitant (1.7\%). Conclusion: This study provides meaningful clinical evidence to help make decisions on HPC use more easily at an appropriate time.

Key Words: Advance directives, Neoplasms, Hospices, Palliative care, Decision trees
Received July 13, 2021

Revised August 1, 2021

Accepted August 2, 2021

\section{Correspondence to}

Kyung-Ah Kang

ORCID:

https://orcid.org/0000-0002-3799-9554

E-mail:kangka@syu.ac.kr

This research is a summary of the first author's master's degree dissertation.

\section{INTRODUCTION}

\section{Background}

In South Korea, cancer survival rates have steadily increased as hospice palliative care (HPC) programs started to be systematized after the enactment of cancer management policy in 2003. The number of HPC specialty institutions expanded to 86 institutions with 1,405 beds as of 2020. Various programs have aimed to improve terminal patients' quality of life [1]. Laws have been implemented to facilitate the provision of
HPC, including the expansion of service recipients to patients with cancer, chronic obstructive pulmonary disease, AIDS, and chronic cirrhosis with the enactment of the Act on Hospice and Palliative Care and Decisions on Life-Sustaining Treatment for Patients at the End of Life (hereinafter referred to as the Act on Decisions on Life-Sustaining Treatment) on August 4, 2017 [2].

Even after the enactment of Act on Decisions on LifeSustaining Treatment in February 2018, important issues have remained in the clinical field (e.g., patients' self-determination) that create difficulties in HPC decision-making in the clinical 
field. Since the enactment of this law, limitations have included insufficient access to HPC services, a lack of infrastructure for decisions regarding the suspension of life-sustaining treatment, unavailability of information about the end of life, and lack of awareness [3]. Moreover, it is difficult to establish patients' desires regarding the suspension of life-sustaining treatment, and it is likely that decisions are made based on speculations about patients' wishes. Patients' autonomy might be overlooked due to caregiver-related economic and emotional factors, including the lack of a sociocultural atmosphere that allows discussions about patients' impending death and the absence of governmental support for hospital fees or public nursing services [4].

In South Korean society, patients' family members and attending physicians-rather than patients themselves-consult to decide on the suspension of life-sustaining treatment. Reasons for this include the sociocultural environment of South Korea, in which discussions about death during patients' sickness are taboo, and aspects of the current clinical environment, wherein it is easier to reach consensus with family members than to obtain decisions from patients [5-7]. According to the medical ethics guideline of the Korean Academy of Medical Sciences, given clinical circumstances wherein referrals to HPC are made after a decision is made to suspend life-sustaining treatment, the timing of the decision to suspend life-sustaining treatment is important to ensure the provision of adequate HPC; however, this process is not always straightforward to follow in practice [8-12]. Therefore, easier-to-use clinical prediction pathways that guide decision-making for HPC would be essential for clinical practice.

A strength of data mining is that it does not require particular statistical assumptions, unlike regression analyses or structural equations, and it provides the foundation for the development of detailed and accurate categorization of the target population as well as prediction and policy [13]. Decision tree model analysis, which is the type of data mining method used in this study, considers the effect of interactions that can occur among variables and produces a prediction model that best explains the dependent variables from large datasets. Therefore, it is useful both for the exploration of decision-making factors and also for the derivation of risk groups or prediction models, making it effective for the analysis of public health-related data [13]. It also takes the rules of creating groups based on theoretical insights from previous studies and practice guidelines and turns them into a tree structure, producing a workflow that is easy to understand and effective for categorization and prediction [14].

This study was conducted to provide preliminary data to assist in the suspension of meaningless life-sustaining treatment and promote timely decisions to use HPC in clinical practice by understanding the impact of related factors through a decision tree model analysis of HPC use by terminal cancer $\mathrm{pa}^{-}$ tients according to their characteristics.

\section{Purpose}

This study aimed to understand HPC use by terminal cancer patients according to their treatment or palliative care-related characteristics in order to facilitate timely decisions to use HPC so that patients can prepare for a meaningful death. The specific goals were as follows:

1) To understand the sociodemographic factors, diseaserelated factors, and types of life-sustaining treatment and palliative care services of terminal cancer patients.

2) To explore differences in HPC use according to terminal cancer patients' sociodemographic factors, disease-related factors, and types of life-sustaining treatment and palliative care services.

3) To understand the predictive factors of HPC use by terminal cancer patients.

\section{METHODS}

\section{Study design}

This retrospective study aimed to understand HPC use according to the treatment or palliative care-related characteristics of terminal cancer patients through a decision tree model analysis. Based on previous studies and clinical guidelines regarding life-sustaining treatment [2-6,8-10,12], patients' sociodemographic, disease-related, and life-sustaining treatment and palliative care service-related characteristics were selected as predictors of terminal cancer patients' decision-making regarding the use of HPC. The conceptual framework of this study is demonstrated in Figure 1. 


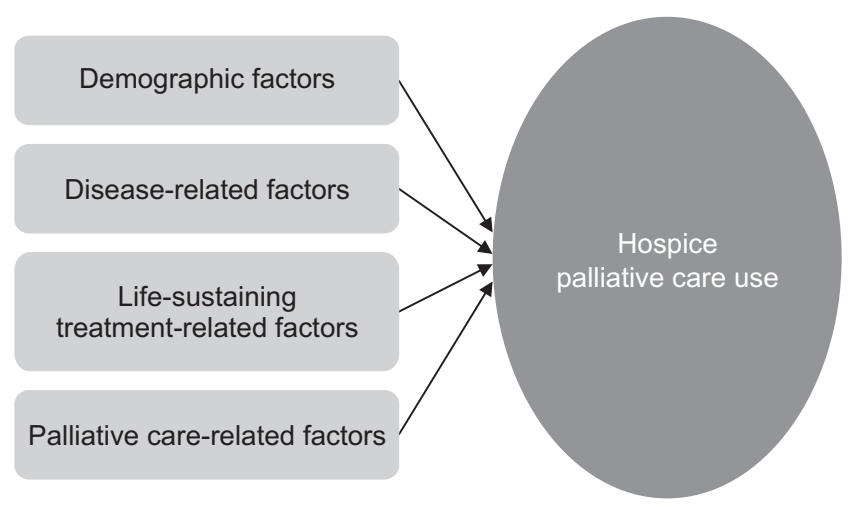

Figure 1. Conceptual framework.

\section{Participants}

The participants were terminal cancer patients who were hospitalized between January 1, 2019 and March 31, 2021 at a cancer specialty hospital located in Seoul, South Korea. The medical records of 394 patients who decided to suspend lifesustaining treatment and completed physician orders for lifesustaining treatment (POLST) were used. Data from patients younger than 20 years of age and incomplete data were excluded.

The minimum number of samples required for a categorization model using data mining is $6 \times$ the number of categories in the outcome variable (2) $\times$ the number of variables (11) [13], which yielded a value of at least 132 . The medical records of 406 patients were collected, but data from 12 patients were excluded according to the above-described criteria. Data from 394 patients were used in the final analysis, exceeding the minimum required sample size for decision tree model analysis.

\section{Study tools}

A decision tree model is a tree structure composed of components referred to as nodes [14]. The aim of research using this framework is to identify appropriate branching from the root nodes, as described below. The target variable (dependent variable, root node) used in this study was the use of HPC, and the independent variables were general characteristics, including participants' sex, age, education, religion, marital status, and illness insight, along with the use of a central venous catheter, oxygenation, total parenteral nutrition (TPN), pain control, and sedation. These predictor variables were selected based on previous studies $[4,15-17]$ to construct a tree structure that would enable identification of the patients who utilized HPC. To test the validity of the tree structure, the team lead of the HPC department, two specialty nurses, one nursing school professor, and one business school professor who specializes in data mining methods were consulted, and the selected study tool went through revisions and updates accordingly.

\section{Data collection}

Data for analysis was extracted from the electronic medical record (EMR) system with cooperation from the medical records team. Demographic information, such as age and sex, and hospitalization-related information, such as diagnosis, pain, respiratory symptoms, alertness, and TPN treatment through a central venous catheter collected from nursing records, drug administration records, and doctors' orders.

\section{Data analysis}

The collected data were analyzed using SPSS version 25.0 (IBM Corp., Armonk, NY, USA).

1) Descriptive statistics (number, percentage, average, and standard deviation) of each measured variable were calculated. Differences in HPC use by sociodemographic characteristics, disease characteristics, and type of life-sustaining treatment and palliative care services were examined using cross-tabs (i.e., with the chi-square test).

2) To identify HPC use groups among terminal cancer $\mathrm{pa}^{-}$ tients, decision tree model analysis was used. The analysis algorithm used chi-square automatic interaction detection to split the decision tree model nodes for HPC use, which was a binary dependent variable [14]. The threshold for splitting and merging of independent variables was 0.05 . Fourteen variables were used as predictors of hospice use: five sociodemographic factors (sex, age, education, religion, and cohabitation), four disease-related factors (illness insight, POLST writing time after diagnosis, period to death after writing POLST, and period to death after diagnosis), and five factors related to lifesustaining treatment and palliative care service (central venous catheter, oxygenation, pain control, TPN, and sedation).

The validity of this study was evaluated by segmenting the observed data [14] into training data and test data at a 50:50 ratio. When the training data and test data were compared, 
the risk estimate in the training data was 0.293 (standard error $0.032)$ and that in the test data was $0.342(0.034)$. This result shows that it is possible to generalize the model of predictive factors for HPC use. The decision tree model analysis was thus conducted with the total data and not the segmented (training and test) data. The maximum depth of the tree was selected at 3 , which is the default value, the minimum number of parent nodes was 50 , and the minimum number of child nodes was 30. The threshold for splitting was determined using the chisquare statistic, and the threshold for statistical significance was $5 \%$.

3) A logistic regression model refers to non-linear regression analysis with quantitative variables as independent variables and a binary variable $(0,1)$ as a dependent variable. In order to analyze the effect of the independent variables, rather than predicting the cases when the event happened (1) or did not happen (0), it predicts the probability of the event occurring [18]. Therefore, this method was used to compare the goodness of fit of decision tree models. Variables that were statistically significant among sociodemographic characteristics and five types of life-sustaining treatment and palliative care services were included in the logistic regression analysis. The cutoff for multicollinearity was a variance inflation factor $<10$, and all the variables satisfied this criterion. Model goodness of fit was evaluated using the Hosmer-Lemeshow test, with a Pvalue $>0.05$.

\section{Ethical considerations}

This study was conducted after receiving approval (202012-005) from the Korea Institute of Radiological and Medical Sciences Institutional Review Board (IRB). According to the approval conditions from the IRB, medical records were analyzed retrospectively with data from patients whose EMR could be accessed, with the exclusion of currently hospitalized patients. To prevent violations of patient privacy, precautions regarding the security of private information were taken, and data were anonymized to make patients impossible to identify.

\section{RESULTS}

\section{Sociodemographic characteristics, disease-related characteristics, and types of life- sustaining treatment of study participants}

The participants' characteristics were as follows (Table 1): 207 were male (52.5\%) and 187 were female (47.5\%), 185 were under the age of $70(47 \%)$ and 209 were 70 or older (53\%), 204 (51.8\%) had an education level of high school graduation or higher, $228(57.9 \%)$ had a religious affiliation, $334(84.8 \%)$ were living with others, 330 (83.8\%) had insight into their illness, 363 (92.1\%) had a central venous catheter inserted, 332 (84.3\%) were oxygenated, 301 (76.4\%) received pain control, and $281(71.3 \%)$ received TPN treatment, and $307(77.9 \%)$ were sedated.

\section{Differences in HPC use according to participants' characteristics}

Significant differences in HPC use were found according to cohabitation $(P<0.001)$, central venous catheter $(P<0.001)$, pain control $(\mathrm{P}<0.001)$, TPN treatment $(\mathrm{P}<0.001)$, and sedation $(\mathrm{P}<0.004)$ (Table 2).

\section{HPC use groups of terminal cancer patients defined by the decision tree model}

The decision tree model analysis included the total sample since the segmented samples did not demonstrate significant differences. The risk estimate was 0.272 (standard error 0.022). The model's prediction accuracy for HPC use was $72.8 \%$. The following factors could be used to identify the group of terminal cancer patients with high HPC use: cohabitation, pain control, and period to death after writing POLST (Figure 2). The group with the highest predicted value for HPC use had cohabitants, was treated for pain, and comprised terminal cancer patients whose death occurred more than 2 months after they wrote POLST. In this group, the probability of HPC use was $87.5 \%$. The group with the second-highest predicted value for HPC use had cohabitants and was treated for pain; $64.8 \%$ of this group opted to use HPC. Lastly, $55.1 \%$ of patients with cohabitants used HPC, which greatly exceeded the $1.7 \%$ proportion of patients without cohabitants. 
Table 1. Characteristics of Study Participants ( $N=394)$.

\begin{tabular}{|c|c|c|c|}
\hline Characteristics & Categories & $\mathrm{n}(\%)$ & $\mathrm{M} \pm \mathrm{SD}$ \\
\hline \multicolumn{4}{|l|}{ Demographic } \\
\hline \multirow[t]{2}{*}{ Sex } & Male & $207(52.5)$ & \\
\hline & Female & $187(47.5)$ & \\
\hline \multirow[t]{2}{*}{ Age (yr) } & $<70$ & $185(47.0)$ & $69.90 \pm 11.33$ \\
\hline & $\geq 70$ & $209(53.0)$ & \\
\hline \multirow[t]{2}{*}{ Education level } & $\leq$ High school & $190(48.2)$ & \\
\hline & > University & $204(51.8)$ & \\
\hline \multirow[t]{2}{*}{ Religious affiliation } & Yes & $228(57.9)$ & \\
\hline & No & $166(42.1)$ & \\
\hline \multirow[t]{2}{*}{ Living together } & Yes & $334(84.8)$ & \\
\hline & No & $60(15.2)$ & \\
\hline \multicolumn{4}{|l|}{ Disease-related } \\
\hline \multirow[t]{8}{*}{ Cancer type } & Lung & $84(21.3)$ & \\
\hline & Liver/bile duct & $58(14.7)$ & \\
\hline & Pancreas & $51(12.9)$ & \\
\hline & Colon & $42(10.7)$ & \\
\hline & Stomach & $36(9.1)$ & \\
\hline & Breast & $12(3.0)$ & \\
\hline & Hematological cancer & $12(3.0)$ & \\
\hline & Other solid & $99(25.1)$ & \\
\hline \multirow[t]{2}{*}{ Recognition of one's illness } & Yes & $330(83.8)$ & \\
\hline & No & $64(16.2)$ & \\
\hline \multirow[t]{2}{*}{ POLST writing time after diagnosis (mo) } & $<30$ & $262(66.5)$ & $30.42 \pm 43.28$ \\
\hline & $\geq 30$ & $115(29.2)$ & \\
\hline \multirow[t]{2}{*}{ Period to death after writing POLST (mo) } & $<2$ & $318(80.7)$ & $1.31 \pm 0.97$ \\
\hline & $\geq 2$ & $60(15.2)$ & \\
\hline \multirow[t]{2}{*}{ Period to death after diagnosis (mo) } & $<30$ & $269(68.3)$ & $32.43 \pm 47.46$ \\
\hline & $\geq 30$ & $124(31.5)$ & \\
\hline \multirow[t]{2}{*}{ HPC use } & Yes & $185(47.0)$ & \\
\hline & No & $209(53.0)$ & \\
\hline \multicolumn{4}{|l|}{ Life-sustaining treatment-related } \\
\hline \multirow[t]{2}{*}{ Central venous catheter } & Yes & $363(92.1)$ & \\
\hline & No & $31(7.9)$ & \\
\hline \multirow[t]{2}{*}{ TPN } & Yes & $281(71.3)$ & \\
\hline & No & $113(28.7)$ & \\
\hline \multicolumn{4}{|l|}{ Palliative care-related } \\
\hline \multirow[t]{2}{*}{ Oxygenation } & Yes & $332(84.3)$ & \\
\hline & No & $62(15.7)$ & \\
\hline \multirow[t]{2}{*}{ Pain control } & Yes & $301(76.4)$ & \\
\hline & No & $93(23.6)$ & \\
\hline \multirow[t]{2}{*}{ Sedation } & Yes & $307(77.9)$ & \\
\hline & No & $87(22.1)$ & \\
\hline
\end{tabular}

POLST: physician orders for life-sustaining treatment, HPC: hospice and palliative care, TPN: total parenteral nutrition.

\section{Logistic regression analysis of the HPC use (test of model fit)}

Logistic regression analysis was conducted to test the good- ness of fit of the model identified using the decision tree model analysis. HPC use was 71.83 times (95\% CI=9.35 552.10) higher among patients with cohabitants than among those with no cohabitants. Patients who received pain control were 
Table 2. Differences in the Use of Hospice Palliative Care according to Participants' Characteristics ( $N=394$ ).

\begin{tabular}{|c|c|c|c|c|c|}
\hline \multirow{2}{*}{ Characteristics } & \multirow{2}{*}{ Categories } & \multirow{2}{*}{$\begin{array}{c}\text { Yes }(n=185) \\
n(\%)\end{array}$} & \multirow{2}{*}{$\frac{\text { No }(n=209)}{n(\%)}$} & \multirow{2}{*}{$\chi^{2}$} & \multirow{2}{*}{$\mathrm{P}$} \\
\hline & & & & & \\
\hline \multirow[t]{2}{*}{ Sex } & Male & $103(26.1)$ & $104(26.4)$ & 1.38 & 0.241 \\
\hline & Female & $82(20.8)$ & $105(26.6)$ & & \\
\hline \multirow[t]{2}{*}{ Age (yr) } & $<70$ & $89(22.6)$ & $96(24.4)$ & 0.19 & 0.666 \\
\hline & $\geq 70$ & $96(24.4)$ & $113(28.7)$ & & \\
\hline \multirow[t]{2}{*}{ Education level } & $\leq$ High school & $91(23.1)$ & $99(25.1)$ & 0.13 & 0.718 \\
\hline & >University & $94(23.9)$ & $110(27.9)$ & & \\
\hline \multirow[t]{2}{*}{ Religious affiliation } & Yes & $108(27.4)$ & $120(30.5)$ & 0.04 & 0.847 \\
\hline & No & $77(19.5)$ & $89(22.6)$ & & \\
\hline \multirow[t]{2}{*}{ Living together } & Yes & $184(46.7)$ & $150(38.1)$ & 58.28 & $<0.001$ \\
\hline & No & $1(0.3)$ & $59(15.0)$ & & \\
\hline \multirow[t]{2}{*}{ Recognition of one's illness } & Yes & $157(39.8)$ & $173(43.9)$ & 0.32 & 0.575 \\
\hline & No & $28(7.1)$ & $36(9.1)$ & & \\
\hline \multirow[t]{2}{*}{ POLST writing time after diagnosis (mo) } & $<30$ & $122(32.4)$ & $140(37.1)$ & 1.73 & 0.189 \\
\hline & $\geq 30$ & $62(16.4)$ & $53(14.1)$ & & \\
\hline \multirow[t]{2}{*}{ Period to death after writing POLST (mo) } & $<2$ & $138(36.5)$ & $180(47.6)$ & 22.37 & $<0.001$ \\
\hline & $\geq 2$ & $46(12.2)$ & $14(3.7)$ & & \\
\hline \multirow[t]{2}{*}{ Period to death after diagnosis (mo) } & $<30$ & $120(30.5)$ & $149(37.9)$ & 2.08 & 0.149 \\
\hline & $\geq 30$ & $65(16.5)$ & $59(15.0)$ & & \\
\hline \multirow[t]{2}{*}{ Central venous catheter } & Yes & $185(47.0)$ & $178(45.2)$ & 29.78 & $<0.001$ \\
\hline & No & $0(0.0)$ & $31(7.9)$ & & \\
\hline \multirow[t]{2}{*}{ Oxygenation } & Yes & $146(37.1)$ & $186(47.2)$ & 7.51 & $<0.006$ \\
\hline & No & $39(9.9)$ & $23(5.8)$ & & \\
\hline \multirow[t]{2}{*}{ Pain control } & Yes & $172(43.7)$ & $129(32.7)$ & 53.15 & $<0.001$ \\
\hline & No & $13(3.3)$ & $80(20.3)$ & & \\
\hline \multirow[t]{2}{*}{ TPN } & Yes & $152(38.6)$ & $129(32.7)$ & 20.04 & $<0.001$ \\
\hline & No & $33(8.4)$ & $80(20.3)$ & & \\
\hline \multirow[t]{2}{*}{ Sedation } & Yes & 156 (39.6) & $151(38.6)$ & 8.32 & $<0.004$ \\
\hline & No & $29(7.4)$ & $58(14.7)$ & & \\
\hline
\end{tabular}

POLST: physician orders for life-sustaining treatment, TPN: total parenteral nutrition.

8.60 times (95\% CI=3.96 18.63) more likely to use HPC than those who did not. Patients who received TPN treatment were 1.92 times (95\% CI=1.06 3.47) more likely to use HPC than those who did not receive TPN treatment. Oxygenated patients were less likely to utilize HPC (OR=0.32; 95\% CI $=0.15 \sim 0.70)$ than those who were not oxygenated. Patients whose death occurred less than 2 months after writing POLST were less likely to utilize $\mathrm{HPC}(\mathrm{OR}=0.20 ; 95 \% \mathrm{CI}=0.87 \sim 0.47)$ than those whose death occurred more than 2 months after writing POLST (Table 3). Based on the above results, the predictor variables identified in the decision tree model were also shown to be significant in the logistic regression analysis.

\section{DISCUSSION}

This study explored the sociodemographic characteristics, disease-related characteristics, and types of life-sustaining treatment and palliative care services received by terminal cancer patients, with the goal of predicting the effect of these factors on HPC use, and identifying preliminary data to increase the quality of life of terminal cancer patients by assisting in the timely use of HPC after writing POLST.

According to the study results, cohabitation had the greatest impact on HPC use. The probability of HPC use of terminal cancer patients with cohabitants was $55.1 \%$, which was significantly greater than the proportion (1.73\%) among terminal cancer patients without cohabitants. Meanwhile, 96 (24.4\%) 


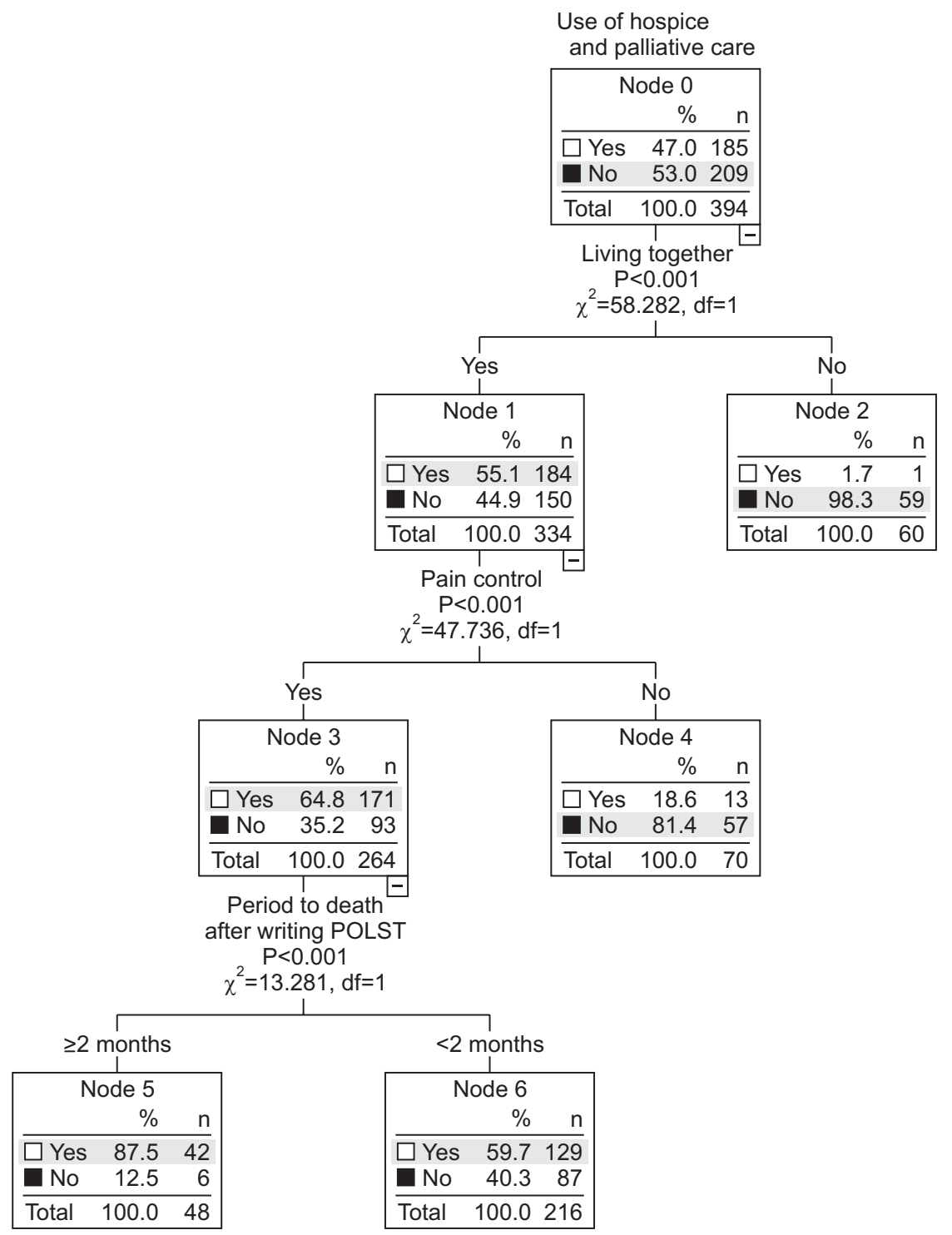

Figure 2. Predictive factors for the use of hospice and palliative care: Decision tree model.

Table 3. Predictive Factors for the Use of Hospice Palliative Care ( $N=394)$.

\begin{tabular}{lrrrcc}
\hline \multicolumn{1}{c}{ Variables } & B & SE & Wald & Odds ratio & 95\% Cl \\
\hline Constant & 24.74 & 7083.08 & - & - & - \\
Living together (yes) & 4.27 & 1.04 & 16.87 & $71.83 *$ & $9.35 \sim 552.10$ \\
Period to death after writing POLST $(<2$ mo) & -1.62 & 0.42 & 14.53 & $0.20 *$ & $0.87 \sim 0.47$ \\
Central venous catheter (yes) & 20.84 & 7083.08 & 0.01 & 1126068778 & 0.00 \\
Oxygenation (yes) & -1.14 & 0.40 & 8.21 & $0.32 *$ & $0.15 \sim 0.70$ \\
Pain control (yes) & 2.15 & 0.40 & 29.67 & $8.60 *$ & $3.96 \sim 18.63$ \\
TPN (yes) & 0.65 & 0.30 & 4.63 & $1.92 *$ & $1.06 \sim 3.47$ \\
Sedation (yes) & 0.14 & 0.34 & 0.16 & 1.14 & $0.58 \sim 2.22$ \\
Model fit $\chi^{2}$ & & & & & $171.07(\mathrm{P}<0.001)$ \\
Hosmer \& Lemeshow $\chi^{2}$ & & & & $4.48(\mathrm{P}=0.612)$ \\
\hline
\end{tabular}

POLST: physician orders for life-sustaining treatment, TPN: total parenteral nutrition.

$* P<0.05$. 
HPC users were aged 70 or older, which exceeded the proportion of those who were under the age of $70(n=89,22.6 \%)$. The preference for use of professional HPC was higher when patients were older. Age was not a significant predictor of HPC use in this study, but a repeated study with a larger sample size is necessary to explore the interactions between the presence of cohabitants and age and to hypothesize predictor variables using the decision tree model.

The second group of HPC users had cohabitants and received treatment for pain control. In this group of patients, $64.8 \%$ utilized HPC. Two earlier studies of terminal cancer patients $[19,20]$ found that patients had the greatest need to control physical symptoms, including pain, followed by their need for emotional support nursing; those findings align with the results of this study. In the study by Nam [21], emotional needs had the highest score (4.45) among the sub-areas of HPC needs. Since the participants in that study had a high ability to carry out their daily routine, it is possible that HPC needs might differ according to disease severity. Future studies should conduct comparative analyses of differences in patients' care needs according to the stage of cancer progression.

The third relevant factor was the time from completion of POLST to death. The probability of HPC use was $87.5 \%$ in terminal cancer patients who had cohabitants, who received pain control, and whose death occurred more than 2 months after writing POLST. In the study by Son and Park [20], the level of HPC needs was highest when the time since cancer diagnosis was less than 1 3 years, and the lowest level of needs was found when the time since diagnosis was more than 3 years, demonstrating that the hospice care needs of cancer patients were significantly different according to the time since diagnosis. In Yeom's study [22], 13,136 (61.3\%) patients out of the total 21,329 studied started using hospice care within 1 year of their diagnosis. It was also reported that the survival time from when patients first started using hospice care was less than 3 months in $94.5 \%$ of patients. Compared to this study's finding that the probability of HPC use was highest when death occurred more than 2 months after writing POLST, it can be inferred that patients at the end of life and advanced cancer patients whose quality of life can depend on symptom management may wish to use HPC at different time points. Further research regarding the timing of use is neces- sary.

Meanwhile, HPC was utilized by fewer than half of the patients who wrote POLST ( $\mathrm{n}=185,47.0 \%)$, and overall, 209 (53.0\%) patients did not utilize hospice and palliative care. This result suggests that the completion of POLST does not necessarily lead to the use of HPC [23,24]. The number of designated HPC institutions has increased to 87 throughout Korea, and the yearly average rate of service use increased from $7.3 \%$ in 2008 to $12.7 \%$ in 2013 and $22.9 \%$ in 2018 [1]. However, to increase HPC use, large-scale education and publicity efforts by the government are necessary. Furthermore, consensus among family members is also an important factor for promoting the use of HPC due to the sociocultural characteristics of South Korea. Generally, in clinical practice in South Korea, terminal status is first notified to family members, who decide whether they will inform the patient $[12,15,25,26]$.

The above-mentioned reasons contribute to the continuation of life-sustaining treatment that the patient does not wish to receive. Therefore, the decision-making process for suspension of treatment in terminal patients should become an interactive decision-making process through logical discussions among medical staff, patient, and family members rather than a onesided notification $[4,7,27,28]$. To maintain quality of life as long as the patient is alive, the transition to HPC at the right moment should be made more effective and easier. Therefore, medical staff should have a detailed guideline for decisionmaking that reflects patients' treatment status in order for $\mathrm{pa}^{-}$ tients to receive HPC after writing POLST $[29,30]$. The results of this study furnish useful information to assist in the smooth transition to HPC use by making it easier to decide to suspend life-sustaining treatment based on POLST completion. These changes will result in the provision of care that helps terminal patients enjoy better quality of life for the rest of their lives and prepare for a comfortable death.

This was a retrospective study that used the medical records of terminal cancer patients who died at one cancer hospital. A limitation is that the analysis did not differentiate the level of oxygenation and TPN provided to patients. Future studies should expand the sample size in order to identify factors of HPC use more clearly. 


\section{CONFLICT OF INTEREST}

No potential conflict of interest relevant to this article was reported.

\section{ORCID}

Hee-Ja Lee, https://orcid.org/0000-0002-8444-5386

Im-Il Na, https://orcid.org/0000-0002-3872-6179

Kyung-Ah Kang, https://orcid.org/0000-0002-3799-9554

\section{AUTHOR'S CONTRIBUTIONS}

Conception or design of the work: HJL, KAK. Data collection: HJL, IIN. Data analysis and interpretation: HJL, KAK. Drafting the article: HJL. Critical revision of the article: HJL. Final approval of the version to be published: HJL, KAK.

\section{SUPPLEMENTARY MATERIALS}

Supplementary materials can be found via https://doi. org/10.14475/jhpc.2021.24.3.184.

\section{REFERENCES}

1. National Cancer Center \& National Hospice Center [Internet]. Goyang: National Cancer Center; 2020 [cited 2021 June 5]. Available from: https://www.hospice.go.kr/index.do.

2. Law on hospice and palliative care utilization and life-sustaining treatment decision making of patients at the end-of-life [Internet]. Sejong: Korean Law Information Center: 2020 [cited 2021 Jun 19] . Available from: http://www.law.go.kr/lsOrdinAstSc.do? tabMenuld=tab2\&quer y=\%EC\%97\%B0\%EB\%AA\%85\%EC\%9D\%98\%EB\%A3\%8C\%EA\%B2\%B0\%EC\%A0\%95\%EB\%B2\%95.

3. Guidance on life-sustaining treatment [Internet] . Sejong: Ministry of Health and Welfare: 2019 [cited 2021 Jun 19] . Available from: http:// www.mohw.go.kr/react/index.jsp.

4. Park SY. Current status of advance care planning and factors affecting in life-sustaining treatment decision-making process for critical ill patients in Korea [dissertation]. Ulsan: Ulsan Univ.; 2018. Korean.

5. Kang NY, Park JY. Clinical characteristics of oncologic patients with DNR decision at a tertiary hospital. Korean J Hosp Palliat Care 2016;19:26-3.

6. Lee JY. The factors that affect the intention to use hospice in terminal cancer patient who take the POLST [master's thesis]. Seoul: Korea Univ.; 2019. Korean.

7. Jang WK, Ahn KG, Kim MY, Kim YR, Han YJ. The role of a patient's family when the withdrawal of life-sustaining treatment is expected: A narrative analysis. Korean J Med Ethics Educ 2018;2:172-89.

8. Han SS, Kim JH, Ku IH, Hong SY, Lee KS. Development of the guideline to Do-Not-Resuscitate (DNR) and ethical review. Korean J Med Ethics Educ 2005;8:116-30.

9. Cappell K, Sundaram V, Park A, Shiraz P, Gupta R, Jenkins P, et al. Advance directive utilization Is associated with less aggressive end-of-life care in patients undergoing allogeneic hematopoietic cell transplantation. Biol Blood Marrow Transplant 2018;24:1035-40.

10. Enguidanos S, Ailshire J. Timing of advance directive completion and relationship to care preferences. J Pain Symptom Manage 2017;53:4956.

11. Miller JJ, Morris P, Files DC, Gower E, Yung M. Decision conflict and regret among surrogate decision makers in the medical intensive care unit. J Crit Care 2016;32:79-84.

12. Son KC. The potential solution for amendments of $\ulcorner$ Act on Life-Sustaining Medical Care Determination $\lrcorner$. Korean Law Association 2020;20:259-87.

13. Bae WS, Cho DH, Seok KH, Kim BS, Choi KL, Lee jE, et al. SAS enterprise miner data mining. Seoul:Kyowoosa;2004.

14. Song TM, Song JY. Social big data research methodology with R. Seoul:Hannarae Publishing Co.;2016.

15. Gwak DY. Hospice palliative care perception and nursing demand of cancer patient's and families [master's thesis]. Busan: Catholic Univ. of Pusan; 2017. Korean.

16. Kim HA. Changes in life-sustaining treatment in terminally ill cancer patients after signing a do-not-resuscitate order. Korean J Hosp Palliat Care 2017;20:93-9.

17. Washington KT, Pike KC, Demiris G, Oliver DP. Unique characteristics of informal hospice cancer caregiving. Supportive Care Cancer 2015;23:2121-8.

18. Kang BS, Kim KS. Social science statistical analysis. Seoul:Hannarae Publishing Co.:2013.

19. Song N, Choi JY. Comparison of the characteristics among Deceased Do-Not-Attempt-Resuscitation (DNAR) cancer patients in hospice and oncology wards. Asian Oncol Nurs 2020;20:10-9. 
20. Son YJ, Park YG. Perception on hospice, attitudes toward death and needs of hospice care between the patients with cancer and family. Korean J Rehabil Nurs 2015;18:11-9.

21. Nam IY. The needs for, knowledge of hospice palliative care and caregiving burden among family member caregivers of patients with cancer [master's thesis]. Seoul: Yonsei Univ.; 2019. Korean.

22. Yeom MS. The effect of hospice system on cancer patients' medical expenses [dissertation]. Seoul: CHA Univ.; 2019. Korean.

23. Hoe MH. A study on the development of the hospice system for the revitalization of life-term care and nursing [master's thesis]. Busan: Pusan Univ.; 2019. Korean.

24. Kang JH, Lee YM, Lee HJ. Effect of the awareness of a good death and perceptions of life-sustaining treatment decisions on attitudes of intensive care nurses toward terminal care. Korean J Critic Care Nurs 2019;12:39-49.

25. Kim GS. A phenomenological study of experiences of family care givers for their terminal cancer patient receiving hospice services at the hospital. Korean J Fam Soc Work 2016;52:35-66.

26. Seo YM, Shin SJ. The relationship among attitudes toward the withdrawal of life-sustaining treatment, death anxiety, and death acceptance among hospitalized elderly cancer patients. Asian Oncol Nurs 2019;19:142-9.

27. Wachterman MW, Pilver C, Smith D, Ersek M, Lipsitz SR, Keating NL. Quality of end-of-life care provided to patients with different serious illnesses. JAMA Intern Med 2016;176:1095-102.

28. Zheng RS, Guo QH, Dong FQ, Owens RG. Chinese oncology nurses' experience on caring for dying patients who are on their final days: a qualitative study. Int J Nurs Stud 2015;52:288-96.

29. Korean Society for Hospice and Palliative Care. Hospice palliative nursing Paju:Koonja;2018. p. 2-29.

30. Sel EM, Koh CK. Critical care nurses' attitudes toward hospice and palliative care and their related factors. Perspectives in Nursing Science 2015;12:94-105. 